\title{
Une étude hydro-sédimentaire globale du littoral vendéen
}

\author{
Amélie ROCHE ${ }^{1}$, Stéphane RAISON ${ }^{2}$ \\ ${ }^{1}$ CETMEF/DELCE, Division Hydraulique et Sédimentologie Maritimes, \\ Technopôle Brest-Iroise, BP 5, 29280 Plouzané, France \\ Amelie.Roche@developpement-durable.gouv.fr \\ ${ }^{2}$ DDE de la Vendée / Service Maritime et des Risques, \\ 1 quai Dingler, BP 10366, 85108 Les Sables d'Olonne Cedex, France \\ Stephane.Raison@developpement-durable.gouv.fr
}

\section{Résumé :}

De nombreuses études hydro-sédimentaires ont été menées sur le littoral vendéen qui est particulièrement exposé aux risques littoraux (submersion marine et érosion). Le manque d'homogénéité et les contradictions entre différentes sources rend difficile l'élaboration de Plans de Prévention des Risques Littoraux cohérents. Les processus côtiers à l'origine de l'aléa érosion ont fait l'objet d'une étude globale au niveau du département. Le groupement de bureaux d'études GEOS \& DHI a utilisé une méthode novatrice de modélisation hydro-sédimentaire afin d'évaluer et de localiser les transits potentiels de manière fiable. Ce travail doit servir de référence pour l'élaboration des futurs PPRL et pour une gestion durable des sédiments en Vendée.

\begin{abstract}
:
Many hydrosedimentary studies were led on Vendée's coasts that are particularly exposed to coastal hazards (flooding and erosion). A lack of homogeneity and contradictions between different sources make it difficult to elaborate coherent prevention plans for coastal hazards. Coastal processes responsible for erosion were studied at a regional scale. The associate research groups GEOS \& DHI used an innovative method for modelling sedimentary fluxes showing in particular global budgets and localisations in an accurate way. This study is expected to be used as a reference for the next elaboration of prevention plans and a sustainable sediment management plan in Vendée.
\end{abstract}

\section{Mots-clés :}

Génie côtier - Érosion - Transport sédimentaire - Gestion durable - Approche globale

DOI: 10.5150/jngcgc.2008.015-R $\quad$ (disponible en ligne - http://www.paralia.fr - available online) 


\section{Introduction : les enjeux du littoral vendéen}

Le littoral vendéen est un territoire marqué par des aménagements lourds réalisés dès l'époque romaine et le Moyen-Age pour gagner des terres agricoles sur la mer en endiguant et asséchant des centaines d'hectares (exemples des marais Breton, Poitevin, etc.). Au cours du $20^{\text {ième }}$ siècle, le développement du tourisme balnéaire a vu la création de nombreuses installations en front de mer. Ces zones poldérisées, aujourd'hui urbanisées, et ces stations balnéaires représentent des enjeux non négligeables notamment en termes de protection civile puisque les unes, situées en dessous du niveau des plus hautes eaux, sont particulièrement exposées à l'aléa de submersion marine alors que les autres subissent également l'aléa d'érosion côtière. La lutte de l'homme contre la mer fait donc entièrement partie de l'histoire vendéenne et explique les nombreux aménagements de défense construits sur son littoral. Ainsi, les $276 \mathrm{~km}$ de côtes vendéennes se décomposent aujourd'hui en environ $40 \%$ de littoral sableux, $40 \%$ de linéaire artificialisé et $20 \%$ de côtes rocheuses (FIERE et al., 2008) (voir figure 1). La tendance générale est à l'érosion mais il existe quelques rares points en accrétion comme le Goulet de Fromentine au Sud de l'Ile de Noirmoutier et la pointe d'Arçay à La Faute sur Mer.

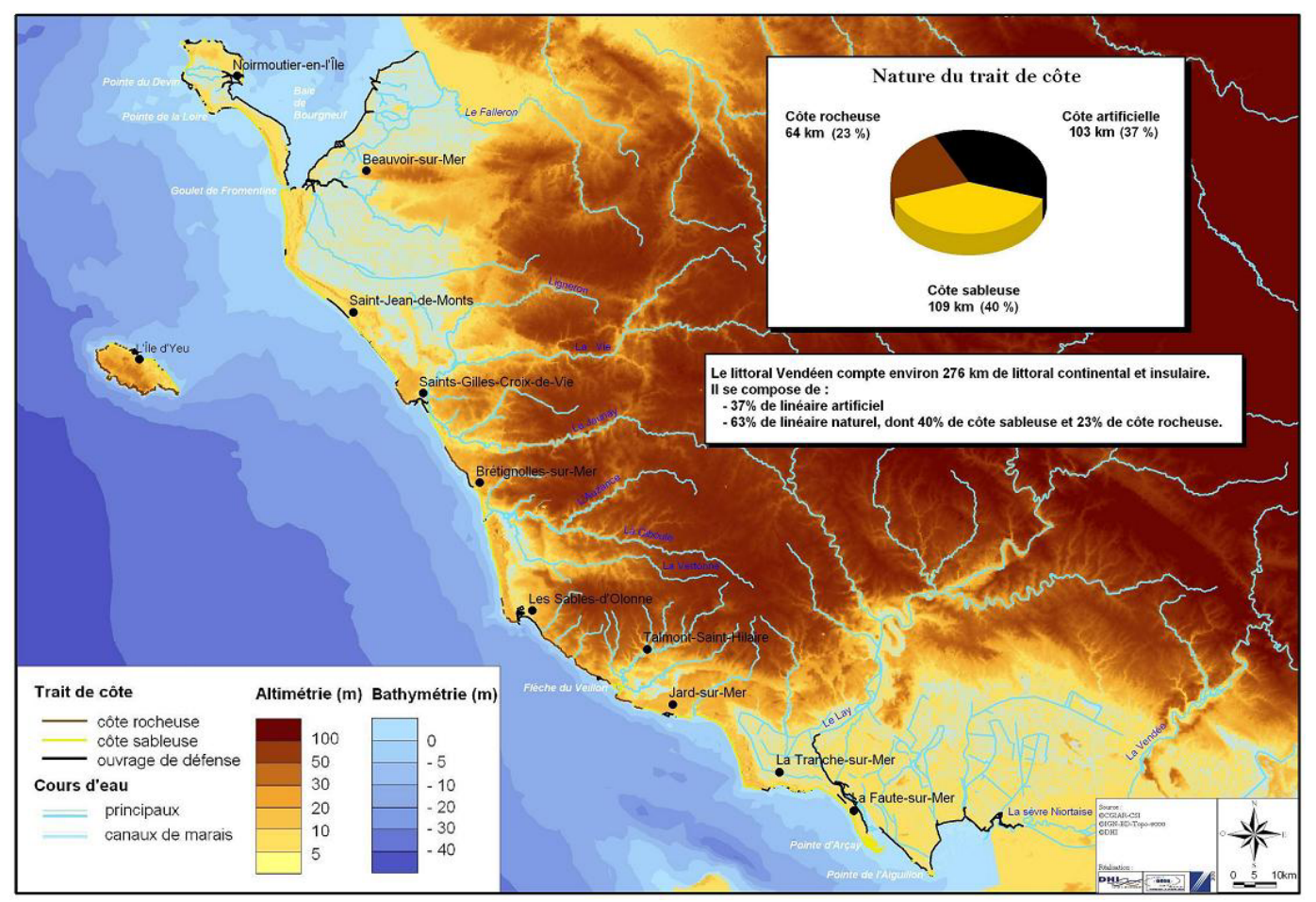

Figure 1. Géographie physique de la Vendée (d'après FIERE et al., 2008)

De nombreuses études hydro-sédimentaires et des collectes de données ont été 
effectuées localement pour des aménagements de défense contre la mer sanstoutefois avoir une approche globale du fonctionnement du littoral et parfois sans véritable cohérence. L'élaboration de Plans de Prévention des Risques Littoraux (PPRL) (MATE \& METL, 1997) vendéens posait donc la question d'une connaissance fine de l'aléa d'érosion et plus précisément du recul du trait de côte (taux de recul, localisation) afin de mettre en place une gestion durable de la ressource en sédiments à travers la valorisation des stocks sédimentaires côtiers disponibles. C'est dans ce contexte que l'« Étude de connaissance des phénomènes d'érosion sur le littoral vendéen" (FIERE et al., 2008) a été réalisée par le groupement de bureaux d'études GEOS et DHI, sous la maîtrise d'ouvrage du MEEDDAT, représenté par le Service Maritime et des Risques de la DDE de la Vendée, et avec l'assistance du CETMEF. Cette étude a fait preuve d'une approche novatrice à plusieurs niveaux et a vocation à servir de référence pour l'élaboration des futurs PPRL et autres études d'aménagement. Dans cet article, l'accent est donc mis sur l'intérêt d'une approche globale et sur la méthodologie de l'étude plutôt que sur la modélisation elle-même des phénomènes, abordée dans FIERE et al. (2008).

\section{Méthodologie définie dans le cadre de l'étude vendéenne}

La connaissance de l'évolution du trait de côte et des principaux processus qui la régissent a été placée au coeur de l'étude. Elle comprenait en outre la connaissance des stocks sédimentaires mobilisables et de leur évolution, la détermination par secteurs des enjeux soumis à l'aléa érosion (avec ou sans submersion) et des solutions possibles compte-tenu de ces enjeux. Une cartographie globale de l'aléa érosion au niveau du département et un inventaire des ouvrages de protection ont également été réalisés.

\subsection{Détermination des unités sédimentaires}

La première étape a consisté en un découpage du littoral en unités sédimentaires indépendantes du point de vue du transport sédimentaire, c'est-à-dire que toute modification du mouvement de sédiments dans une unité doit être sans conséquence directe sur les unités voisines. Il est ainsi possible d'effectuer des bilans sédimentaires au sein de chaque cellule et d'en déduire les éventuels taux d'érosion ou d'accrétion.

Pour ce faire, une carte de la géographie physique de la Vendée (voir figure 1) a été réalisée à partir de la base de données SIG de la DDE, comprenant la topographie, la bathymétrie et l'hydrographie du département. Cette base de données comprenait également l'ensemble des ouvrages de protection répertoriés sur le littoral. Elle s'est révélée être un moyen extrêmement utile pour l'analyse 
morphologique du trait de côte et le découpage en unités sédimentaires.

Un redécoupage de ces unités a ensuite été réalisé pour les besoins de la modélisation numérique du transport sédimentaire. Ce travail s'est essentiellement appuyé sur l'analyse des différentes typologies du littoral (cordons dunaires et flèches sableuses, côtes rocheuses, zones de marais, rivage artificialisé) et sur l'interaction de ces formations avec les embouchures des fleuves, estuaires et havres (souvent synonymes d'apports fluviatiles en sédiments). Une certaine homogénéité des contours bathymétriques a également été recherchée afin de satisfaire l'hypothèse de linéarité du modèle (ici LITDRIFT, voir 2.3.2). Sur le littoral vendéen, sept unités sédimentaires ont pu être distinguées (voir figure 2), avec en général au moins trois sous-unités chacune (excepté pour l'unité sédimentaire de l'Ile d'Yeu qui n'en a qu'une seule).

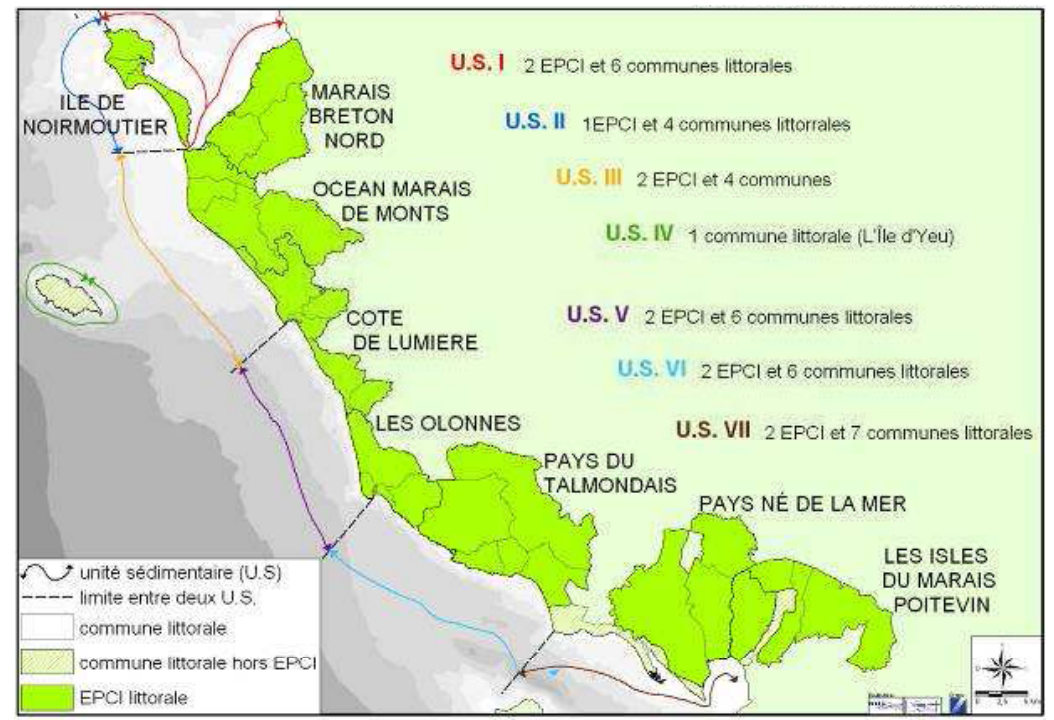

Figure 2. Les 7 unités sédimentaires indépendantes (FIERE et al., 2008)

\subsection{Caractérisation des agents morphodynamiques}

Les agents morphodynamiques (météorologiques et océaniques) ont été répertoriés et cartographiés. Plus que l'action mécanique du gel ou des eaux de ruissellement, c'est l'action du vent qui a été retenue comme paramètre météorologique déterminant. En effet, le vent joue un rôle important dans le transport $\mathrm{du}$ sable directement, mais aussi dans la formation des surcotes météorologiques et de la houle. Le niveau d'eau, la houle et le clapot sont, pour leur part, les facteurs océaniques prépondérants pour le transport sédimentaire à la côte, bien plus que les courants de marée dont l'action se limite au transport des sédiments fins dans les zones particulièrement abritées du littoral vendéen (notamment en Baie de Bourgneuf et de l'Aiguillon). 


\subsection{Caractérisation de l'aléa érosion par modélisation numérique}

Afin d'obtenir une estimation des transits sédimentaires, un couplage de modèles numériques successifs a été réalisé, donnant une modélisation en deux temps.

\subsubsection{Modélisation numérique des états de mer en proche côtier}

Une propagation de la houle du large vers la côte a été réalisée à l'aide du logiciel MIKE 21 SW (DHI, 2007a), modèle de houle spectrale basé sur la conservation de la densité d'action d'onde, prenant en compte les phénomènes de réfraction, de frottement sur le fond, de shoaling et de déferlement. Cette première modélisation a permis d'obtenir une climatologie complète de houle en proche côtier sur 21 ans au pas de temps horaire grâce à l'utilisation de trois points de la base de données ANEMOC, Atlas Numérique des États de Mer Océanique et Côtier, gérée par le CETMEF (BENOIT \& LAFON, 2004). Cet atlas de données de houles le long de la Manche et de l'Atlantique a été réalisé dans le cadre d'une collaboration entre EDF R\&D/LNHE et le CETMEF. La figure 3 présente l'emprise du modèle réalisé avec l'exemple de la propagation des houles d'Ouest.

Les données de forçage du modèle comprennent la houle (hauteur significative spectrale, période pic, direction moyenne, et étalement directionnel de la houle) au pas de temps horaire, issue d'ANEMOC, et le vent, issu des données de vent reconstitué de la base de données AES40 (SWAIL et al., 2000) au large de l'Ile de

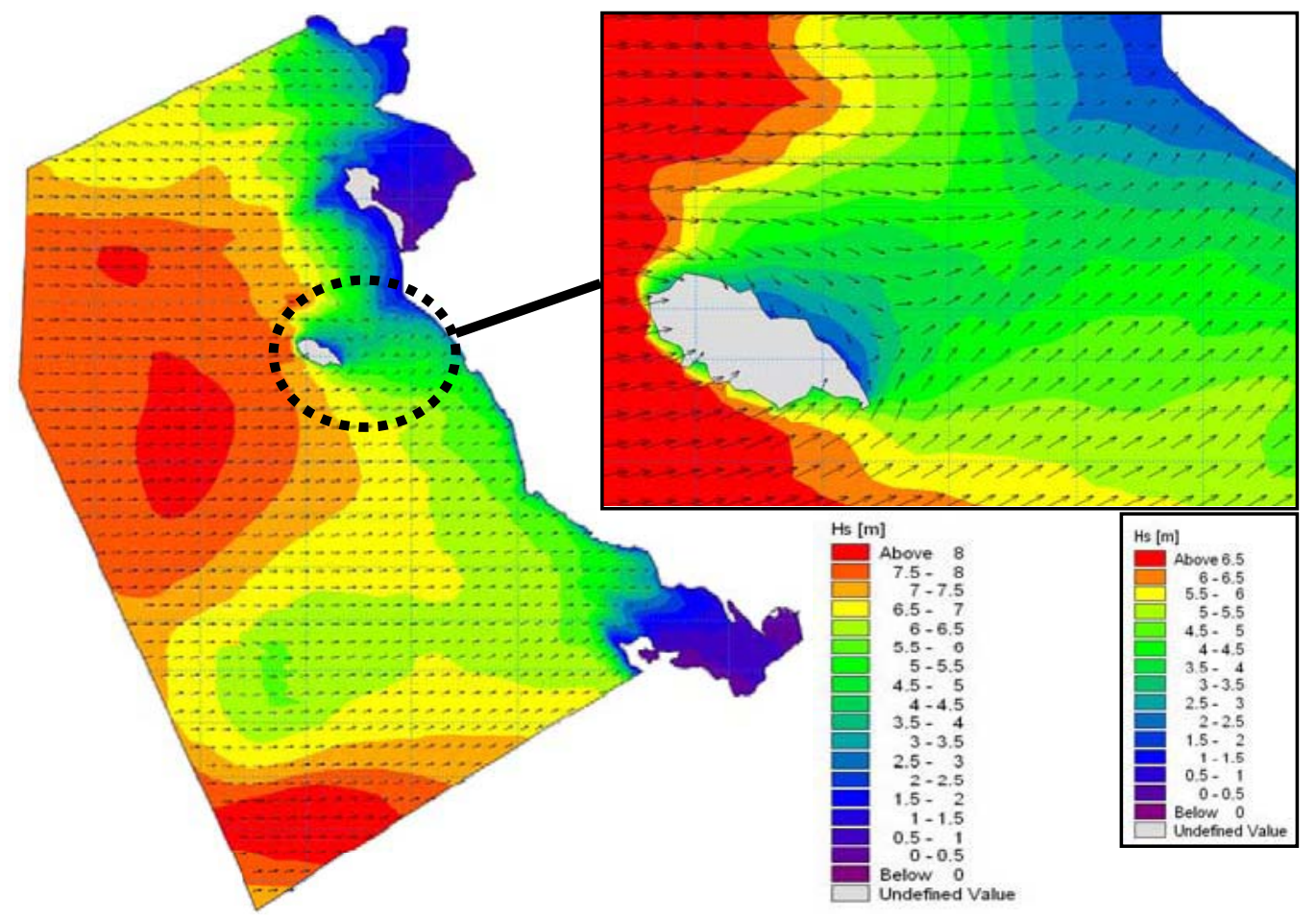

Figure 3. Emprise du modèle vendée et propagation spectrale des houles d'Ouest (hauteurs significatives et direction des houles) (FIERE et al., 2008) 
Noirmoutier au pas de temps 6h. L'évolution temporelle du niveau d'eau est prise en compte en appliquant sur tout le modèle la marée astronomique aux Sables d'Olonne. La bathymétrie est issue de la base de données CMap regroupant les cartes marines du SHOM digitalisées.

Le modèle a été calé par comparaison des données du houlographe de l'Ile d'Yeu sur l'année 1999. A l'issue de cette modélisation, les données de houle ont été extraites en face de chaque sous-unité sédimentaire de façon à servir de données d'entrée au modèle de transport sédimentaire.

\subsubsection{Evaluation des capacités de transport sédimentaire}

Pour obtenir la capacité de transport sédimentaire pour chaque sous-unité, un modèle de transport sédimentaire à une dimension, le module LITDRIFT (DHI, 2007b) du modèle mathématique LITPACK, a été utilisé. Ce modèle prend en compte les effets du frottement sur le fond, du shoaling, du déferlement et du setup. Les données d'entrée du modèle sont un profil et une granulométrie moyenne caractéristiques de la plage et les données de houle en proche côtier. Le transport total par charriage et suspension est alors évalué par le modèle.

Dans cette étude, les houles extraites ont été synthétisées en événements statistiques ramenés à une année et les simulations ont été effectuées en faisant varier l'orientation du trait de côte par pas de $5^{\circ}$ afin d'en déduire les capacités de transports brut (somme des contributions toutes directions confondues) et net (résultante des contributions) de chaque sous-unité sédimentaire. Ainsi, on obtient deux graphes à exploiter, à savoir la distribution dans le profil des transports brut et net avec leurs taux de transport respectifs (pour une orientation donnée) et une courbe donnant les taux de capacité des transport bruts et nets en fonction de l'orientation du trait de côte (donnant également une indication d'« orientation d'équilibre » du trait de côte). Deux graphes sont donnés à titre d'exemple en figure 4 a) et 4 b) pour une plage située en face de Saint-Gilles-Croix-de-Vie. La figure 4 indique une capacité saturée de transport net de 100000 à $200000 \mathrm{~m}^{3} / \mathrm{an}$ pour un transport brut de 200000 à $250000 \mathrm{~m}^{3} / \mathrm{an}$ (a) et un transport sédimentaire se faisant essentiellement dans les fonds inférieurs à $-2 \mathrm{~m}(\mathrm{~b})$.

L'analyse de photographies aériennes géoréférencées a permis d'évaluer les taux de recul du trait de côte entre 1975 et 2001, moyennant des erreurs de précision introduites par la scannérisation des images. La limite de la végétation a servi d'indicateur de position du trait de côte et l'erreur de précision en planimétrie est estimée à $10 \mathrm{~m}$ environ. Les volumes mis en jeu sont calculés par hypothèse de la conservation du profil de plage dans le temps et le long de la plage considérée. Le modèle a ainsi été calé grâce aux taux de recul moyens obtenus sur l'unité sédimentaire 5 et validé par comparaison sur les autres unités sédimentaires. 

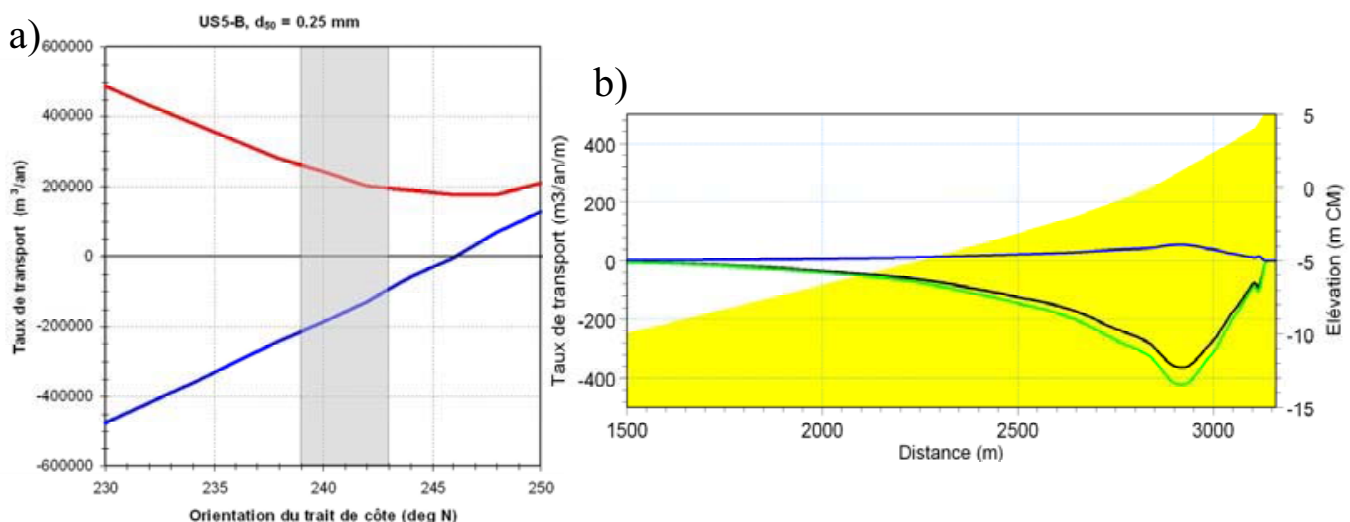

Figure 4. Modélisation du transport sédimentaire au niveau de Saint-Gilles-Croix de-Vie (unité US5-B) : a) Capacité de transport en fonction de l'orientation du trait de côte (transport brut en rouge et net en bleu) ; b) Distribution du transport dans le profil pour une orientation du trait de côte de $240^{\circ}$ (transport net en noir, transport vers le Sud en vert et vers le Nord en bleu). (FIERE et al., 2008)

\subsection{Synthèse de la dynamique littorale globale}

Un bilan sédimentaire est alors effectué sur chaque unité afin de dégager les secteurs stables, en accrétion ou en érosion. L'analyse met en parallèle les gradients de taux d'érosion le long de la cellule sédimentaire et les caractéristiques de la côte (par exemple la présence d'ouvrages ou de flèches rocheuses interrompant une partie du transit sédimentaire). On peut ainsi entre autre estimer les volumes de sédiments qui sortent de la cellule et sont « perdus » au large. Les taux d'érosion obtenus peuvent ensuite être cartographiés et mis en parallèle avec les différents enjeux présents sur le territoire.

\subsection{Cartographie des aléas et des enjeux à l'aide d'un SIG}

Les enjeux socio-économiques et environnementaux touchant le littoral vendéen ont été comparés aux secteurs en érosion mis en avant par la modélisation. L'outil SIG a de nouveau été mis à profit et a permis de synthétiser et de recouper les informations en matière d'aléa d'érosion et de submersion (étude SOGREAH en 2001) avec les enjeux du littoral. Il a ainsi permis de présenter de manière claire et efficace tous les éléments nécessaires à la définition des risques, étape importante dans le processus de mise en place des futurs PPRL locaux.

\section{Une approche globale innovante}

L'intérêt d'une telle démarche réside en premier lieu dans la définition préalable d'un périmètre d'étude à l'échelle des enjeux d'une gestion intégrée du trait de côte. Le second aspect innovant se traduit dans l'utilisation intelligente d'outils numériques, que ce soit un couplage de modèles de propagation de houle et de 
transport sédimentaire ou un SIG, mis au service de l'appui à la décision. Les processus de calage et de validation des résultats modélisés sont également des étapes importantes de certification de la méthode.

\subsection{Une approche « globale » pour une gestion durable des sédiments}

La démarche globale d'une réflexion menée à grande échelle sur la gestion des sédiments n'est peut-être pas totalement nouvelle, pourtant rares sont les services français à avoir entrepris une telle réflexion. Un projet de gestion dynamique des sédiments à l'échelle d'un département avait en effet déjà été lancé par exemple par le Conseil Général de Charente Maritime dès 1998 (PROUST, 2004). L'idée d'un «schéma global de gestion des sédiments » provenait de l'observation de zones d'accumulation sableuse particulièrement importantes non exploitées, parfois même gênantes pour l'activité économique du littoral, alors que certaines côtes sableuses accusaient un déficit sédimentaire. Un système cohérent de transfert de sable paraissait intéressant à développer, notamment en utilisant la technique de rechargement de plage ou de rétablissement artificiel des transits sédimentaires. La démarche avait conduit dans un premier temps à la description au niveau départemental de l'ensemble des transports sédimentaires sableux sur les fonds et à la côte et à la détermination de cellules sédimentaires (étude réalisée par le CETMEF en 2000).Des études plus approfondies avaient ensuite été engagées au niveau local des cellules ainsi définies permettant l'élaboration d'un plan global de gestion des ressources sédimentaires.

Cette approche novatrice est appuyée en 2004 par l'étude « Vivre avec l'érosion côtière en Europe : Espaces et sédiments pour un développement durable » menée dans le cadre du projet européen EUROSION, dont les conclusions prônent une gestion durable des sédiments. L'introduction dans la législation européenne du concept d'« état favorable des sédiments » des zones côtières et l'idée d'un «statut de sédiment utile» dans des zones désignées comme "réserves stratégiques de sédiments » étaient proposées. Les «Plans de Gestion des Sédiments Côtiers » (PGSC) seraient les instruments d'une gestion optimale des sédiments au sein de la Stratégie des Sols de l'Union Européenne pour les zones côtières vulnérables. Il s'agissait aussi de faire reconnaître le rôle des bassins-versants dans le stock sédimentaire et la qualité des sédiments à l'intérieur de la cellule côtière.

Lancée fin 2006, «l'étude de connaissance des phénomènes d'érosion sur le littoral vendéen » a permis d'innover au regard d'autres opérations de gestion du trait de côte en s'inspirant notamment de l'exemple danois (MANGOR, 2004). La valorisation possible des sédiments dragués chaque année au niveau du Goulet de Fromentine, représentant un volume de l'ordre de $100000 \mathrm{~m}^{3} / \mathrm{an}$, dans des secteurs en déficit sédimentaire fait partie des pistes de réflexion engagées. 


\subsection{L'apport de la modélisation et de l'outil SIG}

En plus de la démarche novatrice menée par la DDE de la Vendée dans le cadre de cette étude, c'est la méthodologie employée par les bureaux d'études GEOS \& DHI qu'il faut souligner, et notamment l'apport des outils numériques mis en oeuvre dans leur étude. Une avancée remarquable vient de la quantification des transits sédimentaires le long des côtes réalisée par modélisation, vérifiable et validée par l'analyse de photographies aériennes. Le couplage entre les modèles de propagation de houle et de transport sédimentaire est à la base de la méthode développée par DHI. Cependant, l'utilisation de la base de données ANEMOC a permis d'obtenir les états de mer en proche côtier sur 21 ans, là où les études de modélisation se contentaient auparavant d'une simple analyse statistique des typologies de houle sur quelques années, dépendant des enregistrements de houle disponibles. La faille éventuelle vient de la synthétisation des données en proche côtier en une année « représentative » du climat de houles afin de réduire le temps de calcul du modèle de transport sédimentaire. Il serait sans doute intéressant d'évaluer l'erreur introduite par ce biais statistique par rapport à une modélisation réalisée avec l'ensemble des données de houle obtenues. De plus, l'utilisation d'une granulométrie simple est une autre limite du modèle qui demande une véritable expertise et connaissance du terrain pour analyser les résultats de la modélisation en fonction de la nature du littoral. La comparaison avec les taux de recul obtenus par l'analyse des photographies aériennes permet toutefois de valider raisonnablement les résultats du modèle sédimentaire. Ainsi des évolutions dans ces deux domaines permettraient sûrement d'arriver à un outil particulièrement performant en termes de modélisation, même si les résultats actuels sont déjà tout à fait satisfaisants.

Enfin, le SIG est l'outil indispensable aujourd'hui pour cartographier les risques littoraux et faire apparaître clairement les zones sensibles. Véritable outil d'aide à la décision, le SIG mis en place dans le cadre de l'étude vendéenne s'est appuyé sur l'expérience de la démarche du PLAGE (Plan Littoral d'Actions pour la Gestion de l'Erosion) sur la Côte d'Opale. Ce document constitue une référence commune à l'ensemble du territoire Côte d'Opale et garantit de la cohérence entre divers documents de planification. Fruit d'une démarche concertée comprenant les services de l'Etat, les collectivités territoriales et les acteurs du littoral concernés, le PLAGE consiste techniquement en un SIG qui permet une capitalisation et une gestion aisées des données et fournit les éléments d'analyse nécessaires à l'élaboration de plans d'aménagement locaux. A noter que le PLAGE a également fait l'objet d'une démarche intégrée, en deux temps, suivant la méthodologie adoptée en Charente Maritime. Ainsi, ces différentes études montrent bien que des outils d'aide à la mise en place d'une gestion intégrée existent. Ils sont sans doute 
aujourd'hui les éléments incontournables pour faciliter une gestion durable des sédiments côtiers.

\section{Conclusion}

La méthodologie mise en avant dans cette étude a réussi à valoriser les apports de la modélisation numérique et d'un SIG dans le cadre d'une démarche intégrée de gestion du trait de côte et de la ressource en sédiment. Cette approche exemplaire est totalement en phase avec une gestion durable des sédiments encouragée au niveau européen. Des améliorations restent possibles au niveau de la modélisation mais aussi au niveau réglementaire avec la traduction des PGSC dans la législation française. Toutefois les expériences présentées dans cet article montrent que des moyens existent pour mettre en place des outils simples d'aide à la décision et pour lutter plus efficacement et durablement contre l'érosion côtière.

\section{Références bibliographiques}

1 BENOIT M., LAFON F. (2004). A nearshore wave atlas along the coasts of France based on the numerical modelling of wave climate over 25 years. EDF R\&D LNHE.

2 Direction Générale de l'Environnement - Commission Européenne (2004). Vivre avec l'érosion côtière en Europe : Espaces et sédiments pour un développement durable. Bilans et recommandations du projet EUROSION.

3 DHI (2007a). MIKE 21 Spectral Wave Module. Scientific Documentation.

4 DHI (2007b). Longshore current and littoral drift. LITDRIFT user guide.

5 FIERE M., LE CORNEC E., RAISON S. (2008). Étude de connaissance des phénomènes d'érosion sur le littoral vendéen. Colloque SHF : «Nouvelles approches sur les risques côtiers "), Paris.

6 MANGOR K. (2004). Shoreline Management Guidelines, DHI Water \& Environnement.

7 Ministère de l'Aménagement du Territoire et de l'Environnement / Ministère de l'Equipement, des Transports et du Logement (1997). Plans de prévention des risques littoraux (PPR), guide méthodologique. La documentation Française.

8 PROUST W. (2004). Gestion intégrée des sédiments sur le littoral Charentais. VIII ${ }^{\text {èmes }}$ Journées Nationales Génie Civil - Génie Côtier, Compiègne, pp 817-829.

9 Syndicat Mixte de la Côte d'Opale (2003). Plan Littoral d'Actions pour la Gestion de l'Erosion.

10 SWAIL V.R., CECCACI E.A., COX A.T. (2000). The AES40 north Atlantic wave reanalysis: validation and climate assessment, $6^{\text {th }}$ International Workshop On Wave Hindcasting and Forecasting, November 6-10, 2000 Monterey, California, USA. 